\title{
Utilization Patterns of a Food Referral Program: Findings from the Mid-Ohio Farmacy
}

\author{
Daniel M. Walker, PhD, MPH, Matthew J. DePuccio, PhD, MS, \\ Jennifer L. Hefner, PhD, MPH, Jennifer A. Garner, PhD, RD, \\ Joshua J. Joseph, MD, MPH, Amy Headings, PhD, RD, and Aaron Clark, DO
}

Introduction: There is limited evidence describing utilization of clinic-based food referral programs intended to support healthy eating for food-insecure patients. To address this gap, this study aims to describe the utilization of the Mid-Ohio Farmacy (MOF). MOF is a partnership between a regional foodbank and local health care providers, including an academic medical center (AMC), that enables referrals of patients that experience food insecurity to a network of participating food pantries.

Methods: This observational study uses data from 2 AMC family medicine clinics that offered the MOF referral from September 2019 to November 2020. Patients who screened positive for food insecurity and had an eligible chronic disease (eg, diabetes, hypertension, obesity) were referred to the MOF. We compared demographic and clinical characteristics of patients that filled their referral (ie, visitors) to those that did not (ie, non-visitors). Among visitors, we also assessed patterns of pantry utilization.

Results: In total, 51\% (164 of 322) of patients referred to the MOF visited a food pantry at least once. Visitors were more likely to be older, have diabetes, and have visited a food pantry before their referral. Patients with uncontrolled hypertension were less likely to visit a food pantry following their referral. Patients that had visited a food pantry before their referral had more visits in total and more produce-specific visits following their referral.

Conclusions: Our results suggest that while the MOF can connect patients to food resources, further attention may be needed to encourage its use among patients who have not previously accessed pantries. (J Am Board Fam Med 2021;34:1174-1182.)

Keywords: Family Medicine, Food Insecurity, Healthy Diet, Ohio, Referral and Consultation, Social Determinants of Health

\section{Introduction}

Food insecurity, or the inability to afford and access nutritionally adequate foods consistently, ${ }^{1}$ may represent 1 of the more tractable nonmedical, health-related social needs that can be addressed in a clinical setting. Food insecurity puts individuals at risk for current and

This article was externally peer reviewed.

Submitted 27 January 2021; revised 13 July 2021; accepted 21 July 2021.

From the Department of Family and Community Medicine, College of Medicine, The Ohio State University, Columbus, $\mathrm{OH}$ (DMW, AC); The Center for the Advancement of Team Science, Analytics, and Systems Thinking (CATALYST), College of Medicine, The Ohio State University, Columbus, OH (DMW, MJD); Department of Health Services Management and Policy, College of Public Health, The Ohio State University, Columbus, $\mathrm{OH}$ (JLH); School of Health and Rehabilitation Sciences, College of Medicine and The John Glenn College of Public Affairs, The Ohio State University, Columbus, $\mathrm{OH}$ (JAG); Division of Endocrinology, Diabetes and Metabolism, Department of Internal Medicine, College of future negative health outcomes and exacerbates health inequities. ${ }^{2}$ Specifically, adults and children who experience food insecurity are at an increased risk for obesity, diabetes, and hypertension. ${ }^{3-6}$

Food pantries are community-based organizations that distribute free food to individuals and their

Medicine, The Ohio State University, Columbus, OH (JJJ); Mid-Ohio Food Collective, Grove City, OH (AH).

Funding: This project was supported by a grant from The Ohio State University Department of Family and Community Medicine Crisafi-Monte Endowment Fund. The content is solely the responsibility of the authors and does not necessarily represent the official views of the sponsor.

Conflict of interests: None.

Corresponding author: Daniel M. Walker, $\mathrm{PhD}, \mathrm{MPH}$, Assistant Professor, Department of Family and Community Medicine, College of Medicine, The Ohio State University, 460 Medical Center Drive, Suite 520, Columbus, OH 43210, Phone: 614-293-2428-0943, Fax: 614-293-2715

(E-mail: Daniel.Walker@osumc.edu). 
families and can serve as a primary or secondary food source for individuals with income below a prespecified threshold (eg, in Ohio, individuals below 200\% of the Federal Poverty Level). ${ }^{7}$ Despite concerns about the capacity of food pantries to address longterm food insecurity, ${ }^{8,9}$ they can provide nutritious foods to individuals who may otherwise not have the means to maintain a health-promoting diet. ${ }^{10}$ Therefore, several innovative approaches have emerged that connect patients assessed as food-insecure in clinical settings to food resources available through community-based organizations. ${ }^{11,12}$ These clinic-based programs typically partner with local foodbanks, food pantries, and food voucher systems to facilitate or incentivize patients' access to nutritious foods. ${ }^{13}$ Existing evidence suggests that programs based in primary care clinics can help address patients' unmet needs-including not only improved affordability of and access to nutritious foods but also housing and medication assistance-and can potentially improve clinical outcomes and reduce costs of care. ${ }^{13}$ However, a recent systematic review of clinic-based food referral programs highlights the lack of research on the characteristics of patients using these resources and their patterns of program utilization. ${ }^{14}$ The absence of research on food referral program utilization patterns remains a noted concern, ${ }^{15}$ and this gap in understanding likely contributes to limited integration of community and health care organization services, especially as it relates to food security programming. ${ }^{16}$

To address this gap, we examined the characteristics of patients that use (or not) a food referral program offered by their family medicine provider. Specifically, we evaluated the Mid-Ohio Farmacy (MOF) program implemented at 2 community-based family medicine practices within an academic medical center (AMC). ${ }^{17}$ Understanding the sociodemographic attributes that are associated with varying levels of program uptake and differing patterns of utilization can inform the design of such interventions and the refinement of existing programming to reach and meet the needs of key patient subgroups toward the achievement of health equity.

\section{Methods}

\section{Mid-Obio Farmacy}

In September 2019, the Department of Family and Community Medicine at a large midwestern AMC joined the MOF in partnership with the Mid-Ohio Food Collective ('Food Collective'). The Food Collective is a regional foodbank that delivers over 170,000 meals daily through 680 agency partnerships. Both the AMC and the Food Collective are in a large metropolitan region in Ohio, with estimates of rates of food insecurity ranging from $16.5 \%$ to $32.2 \% .^{18,19}$

The goal of the MOF is to establish a systematic screening and referral process aimed at addressing food insecurity through an electronic referral that connects patients to fresh produce through a food pantry near their homes. The MOF uses a variation on food vouchers: a referral from a patient's primary care provider to 1 of 16 Food Collective partner food pantries in the metropolitan area. All income-eligible visitors to a food pantry can receive produce once a month, but patients referred through the MOF are eligible to receive produce once a week, or 4 times more frequently.

\section{Study Design}

This retrospective observational study of the MOF took place between September 2019 and November 2020. This study was approved by the Ohio State University Institutional Review Board after expedited review.

\section{Screening to Identify MOF Participation}

The sample of patients included in this study presented for primary care at 1 of 2 family medicine clinics offering the MOF referral, both of which are located in urban neighborhoods with lower socioeconomic status and actively screen patients for food insecurity. Patients eligible for MOF referral had to be aged 18 years or older; screen positive for food insecurity; and meet clinical criteria for poor health outcomes as defined by an International Classification of Diseases, Tenth Revision (ICD-10) Clinical Modification based diagnosis of diabetes, type 1 or 2 (ie, ICD-10 E10, E11, E13), ${ }^{20}$ obesity (body mass index $[\mathrm{BMI}] \geq 30 \mathrm{~kg} / \mathrm{m}^{2}$ ) (that is, ICD-10 Z68.3[6 to 9], Z58.4[1 to 5], E66.[1239], or hypertension (ie, ICD10 I10); uncontrolled diabetes defined as a hemoglobin A1C (HbA1C) value $>9 \%$ with or without a diabetes diagnosis; uncontrolled hypertension defined as blood pressure $(\mathrm{BP})>140 / 90$ with a hypertension diagnosis; ${ }^{21}$ or pregnant patients who have gestational diabetes identified from either the problem list or an ICD-10 code of O24.4. 
Food insecurity was assessed, either in-person or during a virtual visit (ie, telehealth), using a validated food insecurity screening tool embedded into the AMC's electronic health record (EHR). Screening occurred on a patient-by-patient basis and was not routinely administered by all providers. The validated screening tool uses 2 questions that are part of the standard United States Department of Agriculture-Economic Research Service 18-item household food security screening module: ${ }^{22,23}$ (1) Within the past 12 months, we worried whether our food would run out before we got money to buy more; (2) Within the past 12 months, the food we bought just did not last, and we did not have money to get more. A response of sometimes true or often true, as opposed to never true, to either statement was considered evidence for risk of food insecurity. For patients who screened positive for food insecurity and met the clinical criteria, a referral to the MOF project could be initiated.

\section{MOF Referral Process}

Patients who met the eligibility criteria for referral to the MOF were asked to sign a release of information (ROI) if the visit was in person or give verbal consent to the ROI if the visit was virtual. The ROI allowed the AMC to transfer patient information about the referral to the Food Collective. Patients that agreed to sign the ROI were mailed a brochure introducing them to the program and identifying participating Food Collective pantry locations. The brochure also included a card with a bar code linked to unique patient identification, the RxID. This card could be presented at any participating pantries to recognize the patient as participating in the MOF program and activate them into the Food Collective's FreshTrak software system. All visits to receive food at pantries were linked to this card and documented in FreshTrak.

\section{Cross-Sector Data Sharing}

Two data sources were merged for the analysis: data from the Food Collective's FreshTrak software system; and data from AMC's EHR. The data sources were merged using the unique RxID recorded in the EHR transferred to the FreshTrak system. The FreshTrak system provides a detailed inventory of the location, date, and frequency of patient visits, patient household size, and the type of food the patient picked up (ie, a produce-specific visit or general food visit). The FreshTrak system also matches patients to encounter records at any Food Collective pantries before the MOF referral using an algorithm that includes patient name and contact information. This information allowed patients and their visits to be characterized based on their prereferral utilization (ie, new or existing visitors; pre- or post-referral visit).

Data from the AMC's EHR included patient demographic information such as age, sex, race (ie, Black, White, Other), insurance status (ie, Medicaid, Medicare, Other), diabetes status, hypertension status, obesity status, and pregnancy status. Other insurance statuses included those with private insurance, self-pay, or unknown insurance status.

\section{Data Analysis}

The aim of this analysis was to characterize utilization patterns of MOF enrollees. To assess the relationship between patient demographics, clinical characteristics, and use of the MOF referral, we compared patients that visited a Food Collective pantry (ie, visitors) following referral to those that did not (ie, non-visitors) using $\chi^{2}$ or $t$ test, as appropriate. We estimated the likelihood of a patient being a visitor to a food pantry using a multivariable logistic regression model controlling for patient demographic (ie, age, sex, race, household size, insurance status, pre-referral food pantry visitor) and clinical characteristics (ie, diabetes, uncontrolled diabetes, hypertension, uncontrolled hypertension, obesity, pregnant), and report the average marginal effect for each characteristic. We then compared new visitors to existing visitors and pre-referral use patterns to post-referral use patterns. Only patients with a minimum of 30 days post-referral data were included in the analytic sample to allow sufficient opportunity to use the MOF. To create comparable time intervals, only patients with a minimum of 90 days post-referral data were included in the analysis of pre- and post-referral use, and all pantry visits beyond 90 days pre- or post-referral were excluded from the analysis.

\section{Results}

Overall, 339 patients were referred to the MOF within the study time frame. Of those, 322 (94\%) agreed to have their information sent from the AMC to the Food Collective. Of the 322 participating patients, a total of 164 (51\%) visited a Food Collective-affiliated food pantry at least once 
Table 1. Characteristics of Patients Referred to and Participating in the Mid-Ohio Farmacy Project

\begin{tabular}{|c|c|c|c|c|}
\hline Characteristic & $\begin{array}{l}\text { Total Referred } \\
\quad(\mathrm{n}=339)\end{array}$ & $\begin{array}{l}\text { Pantry Visitors } \\
\quad(\mathrm{n}=164)\end{array}$ & $\begin{array}{l}\text { Non-Pantry Visitors } \\
\quad(\mathrm{n}=158)\end{array}$ & $\begin{array}{l}\text { Declined } \\
(\mathrm{n}=17)\end{array}$ \\
\hline Mean age (SD) & $51.6(12.9)$ & $53.5(11.5)$ & $49.4(13.6)$ & $54.6(16.0)$ \\
\hline Female, n (\%) & $261(76.9)$ & $130(79.3)$ & $119(75.3)$ & $12(70.6)$ \\
\hline \multicolumn{5}{|l|}{ Race, n (\%) } \\
\hline White & $95(28.0)$ & $44(26.8)$ & $42(26.6)$ & $9(52.9)$ \\
\hline Black & $225(66.4)$ & $112(68.3)$ & $106(67.1)$ & $7(41.2)$ \\
\hline Other & $19(5.6)$ & $8(4.9)$ & $10(6.3)$ & $1(5.9)$ \\
\hline Mean household size (SD)* & $2.4(2.0)$ & $2.9(2.2)$ & $1.9(1.7)$ & - \\
\hline \multicolumn{5}{|l|}{ Insurance, n (\%) } \\
\hline Medicaid & $152(44.8)$ & $73(44.4)$ & $72(45.6)$ & $7(41.2)$ \\
\hline Medicare & $105(30.9)$ & $57(34.7)$ & $44(27.8)$ & $4(23.5)$ \\
\hline Other** & $82(24.2)$ & $34(20.7)$ & $42(26.6)$ & $6(35.3)$ \\
\hline Diabetes, n (\%) & $157(46.3)$ & $86(52.4)$ & $65(41.1)$ & $6(35.3)$ \\
\hline Uncontrolled diabetes, n (\%) & $42(19.3)$ & $25(21.7)$ & $17(18.3)$ & 0 \\
\hline Hypertension, n (\%) & $184(54.3)$ & $91(55.5)$ & $83(52.5)$ & $10(58.8)$ \\
\hline Uncontrolled hypertension, n (\%) & $93(28.1)$ & $32(20.0)$ & $53(34.4)$ & $8(47.1)$ \\
\hline Obesity, n (\%) & $275(81.1)$ & $138(84.1)$ & $123(77.8)$ & $14(82.3)$ \\
\hline Pregnant, n (\%) & $4(1.2)$ & $2(1.2)$ & $1(0.6)$ & $1(5.9)$ \\
\hline Pre-referral visitor, $\mathrm{n}(\%)^{*}$ & $128(39.7)$ & $100(60.0)$ & $28(17.7)$ & - \\
\hline
\end{tabular}

SD, standard deviation.

*Data not available for patients that Declined ( $\mathrm{n}=322)$.

${ }^{* *}$ Other insurance includes patients with private insurance $(\mathrm{n}=70)$, self-pay $(\mathrm{n}=7)$, and unknown $(\mathrm{n}=5)$.

following the referral. Table 1 shows characteristics of referred patients broken down into 3 subgroups: pantry visitors, non-pantry visitors, and those who declined referral.

\section{Pantry Visitor Characteristics}

The results from a multivariable logistic regression model of patient characteristics associated with the use of a Food Collective-affiliated food pantry are shown in Table 2. The marginal effects from this model show that older patients, patients with diabetes, and patients who previously visited a participating food pantry were more likely to visit a participating food pantry following referral. In contrast, patients with uncontrolled hypertension were less likely to visit a food pantry following referral.

\section{Utilization Differences between New and Existing Visitors}

A comparison of utilization between new pantry visitors $(\mathrm{n}=64)$ and patients who had visited a participating food pantry before their MOF referral $(\mathrm{n}=100)$ is presented in Table 3 . New visitors had a significantly lower mean number of total visits over the 90-day studied period. This result held when looking at each month post-referral; new visitors had lower mean visits in the 30-day period, the 30- to 60-day period, and the 60- to 90-day period following referral. New visitors also had a significantly higher mean number of days following referral to their first visit to a participating food pantry, as well as a greater distance traveled to the food pantry. However, no differences were observed in the mean number of produce-specific visits.

\section{Utilization Pre- and Post-Referral}

To examine differences in utilization of the food pantry pre- and post-referral, we compared utilization for all patients that had at least 1 food pantry visit in the pre-referral period. Also, we had at least 90 days of post-referral data available $(\mathrm{n}=81)$ (see Table 4). In the post-referral period, patients had a greater mean total visit count. No differences in the number of food pantry visits were observed within the 30 days post- versus pre-referral, but patients had a greater number of visits in the 30 to 60 -day window post- versus pre-referral. No differences were observed between 60 and 90 days post- and pre-referral. Patients also had a greater number of produce-specific visits in the post-referral period. 
Table 2. Likelihood of Visiting a Food Pantry Among Patients Participatingin the Mid-Ohio Farmacy $(\mathbf{n}=322)$

\begin{tabular}{lccc}
\hline Characteristic & Marginal Effect $(\%)^{*}$ & $95 \%$ CI & $P$ value* \\
\hline Age (1-year) & 0.7 & 0.00 to 0.01 & $<.001$ \\
Female & -0.2 & -0.12 to 0.11 & .96 \\
Race & Ref & Ref & Ref \\
White & -4.6 & -0.15 to 0.06 & .39 \\
Black & -8.8 & -0.31 to 0.13 & .43 \\
$\quad$ Other & 2.6 & -0.00 to 0.05 & .05 \\
Household size & & & Ref \\
Insurance & Ref & -0.14 to 0.09 & .69 \\
$\quad$ Other & -2.4 & -0.19 to 0.07 & .39 \\
$\quad$ Medicaid & -5.9 & 0.01 to 0.21 & .03 \\
$\quad$ Medicare & 11.0 & -0.18 to 0.12 & .70 \\
Diabetes & -3.0 & -0.07 to 0.13 & .57 \\
Uncontrolled diabetes & 2.8 & -0.30 to -0.08 & $<.001$ \\
Hypertension & -19.3 & -0.07 to 0.17 & .45 \\
Uncontrolled hypertension & 4.7 & -0.16 to 0.81 & .19 \\
Obesity & 32.3 & 0.28 to 0.43 & $<.001$ \\
Pregnant & 35.8 & &
\end{tabular}

CI, confidence interval; Ref, reference.

*Average marginal effect as determined via a multivariable regression model.

\section{Discussion}

Clinic-based food referral programs offer family medicine providers an opportunity to address a nonmedical, health-related social need with relevance to their patients' diet-related health conditions: their food insecurity status. While some evidence suggests that these programs may offer health benefits, ${ }^{14}$ little attention has been paid to understanding patient utilization of such referral programs. Our study addresses this gap by reporting on the patient characteristics associated with uptake of a family medicine-based food referral program, as well as provide insight into the program utilization patterns of participating patients.

Our findings reveal that, beyond (older) age, diabetic diagnosis, and uncontrolled hypertension status, a patient's prior use of the Food Collective's pantry network is the factor most associated with their use of the referral program. These patients may have greater access to the food pantries, ${ }^{24}$ or may feel more comfortable and familiar with accessing these resources. Our results also suggest that prior users may access the food pantries more

Table 3. Utilization of Mid-Ohio Farmacy Food Pantries by New and Existing Visitors

\begin{tabular}{|c|c|c|c|c|}
\hline Characteristic & $\begin{array}{c}\text { Total Visitors }(\mathrm{n}=164) \\
\text { Mean }(\mathrm{SD})\end{array}$ & $\begin{array}{c}\text { New Visitors }(\mathrm{n}=64) \\
\text { Mean }(\mathrm{SD})\end{array}$ & $\begin{array}{c}\text { Existing Visitors }(\mathrm{n}=100) \\
\text { Mean }(\mathrm{SD})\end{array}$ & $P$ value* \\
\hline Total visits & $7.8(9.7)$ & $4.0(5.6)$ & $10.1(10.9)$ & $<.001$ \\
\hline Days to first visit & $51.4(75.1)$ & $74.8(97.2)$ & $36.3(51.9)$ & $<.001$ \\
\hline Distance traveled (miles) & $3.7(2.7)$ & $4.4(2.8)$ & $3.3(2.6)$ & .015 \\
\hline Produce visits ${ }^{\dagger}$ & $1.2(3.9)$ & $1.2(2.7)$ & $1.2(4.5)$ & .979 \\
\hline Visits within 30 days post-referral & $1.1(1.5)$ & $0.8(0.9)$ & $1.4(1.6)$ & .012 \\
\hline Visits $>30 \leq 60$ days post-referral & $1.3(1.9)$ & $0.65(1.2)$ & $1.8(2.2)$ & $<.001$ \\
\hline Visits $>60 \leq 90$ days post-referral & $0.9(1.5)$ & $0.5(0.9)$ & $1.2(1.7)$ & .001 \\
\hline
\end{tabular}

$\mathrm{SD}$, standard deviation.

${ }^{*} P$ value estimated from $t$ test.

${ }^{\dagger}$ Produce visits are defined as those where the patient predominantly selects produce items from the food pantry as indicated in the FreshTrak system by pantry staff. 
Table 4. Comparison of Use Pre- and Post-Referral Among Existing Food Pantry Visitors for Users with at Least 90 Days of Post-Referral Data

\begin{tabular}{|c|c|c|c|}
\hline Characteristic & $\begin{array}{c}\text { Pre-Referral }(\mathrm{n}=81) \\
\text { Mean }(\mathrm{SD})\end{array}$ & $\begin{array}{c}\text { Post-Referral }(\mathrm{n}=81) \\
\text { Mean }(\mathrm{SD})\end{array}$ & $P$ value* \\
\hline Produce visits $^{\dagger}$ & $0.5(1.2)$ & $0.9(2.3)$ & .021 \\
\hline Visits within 30 days pre-/post-referral & $1.1(1.6)$ & $1.3(1.6)$ & .201 \\
\hline Visits $>30 \leq 60$ days pre-/post-referral & $0.9(1.2)$ & $1.8(2.2)$ & $<.001$ \\
\hline Visits $>60 \leq 90$ days pre-/post-referral & $1.0(1.6)$ & $1.3(1.6)$ & .251 \\
\hline
\end{tabular}

$\mathrm{SD}$, standard deviation.

${ }^{*} P$ value estimated from $t$ test.

${ }^{\dagger}$ Produce visits are defined as those where the patient predominantly selects produce items from the food pantry as indicated in the FreshTrak system by pantry staff.

following the referral from their family medicine provider than they did before the referral. There is a multitude of possible explanations for this finding. The referral may leverage pre-existing interest in and acceptability of accessing the food pantry network, acting as a reminder, or a nudge, for pantryexperienced patients. ${ }^{25}$ The medical provider's central role in the referral may strengthen patient understanding of and commitment to the connection between food and their health and may influence patients to behave as they would if prescribed a pharmacological intervention. For instance, it is possible that when making a referral to a food pantry, providers used this opportunity to discuss the role of healthy eating in managing and reducing the risk factors associated with diabetes, which may have encouraged these patients to visit a participating food pantry and benefit from the improved access to produce. Finally, our finding that new visitors may have to travel a greater distance to food pantries suggests that community-level factors, including the co-occurrence of other non-medical, social needs, might impact a patient's ability to access the pantries following a referral. Addressing multiple nonmedical, social needs simultaneously may be 1 approach to increase utilization of specific programs. Referrals to the Agency for Healthcare Research and Quality's community hubs and the integration of community health workers into clinical practice are 2 emerging approaches for addressing multiple nonmedical, social needs concurrently. ${ }^{26,27}$

Notably, our findings suggest that the MOF increased the number of produce-specific visits to food pantries for patients with prior pantry visits than before their referral. However, the difference between the mean produce visits and mean total visits suggests that patients may generally receive food from the food pantries rather than produce exclusively. This finding represents an important area of future inquiry for evaluation of the MOF and similar interventions; greater utilization of food pantries related to referral programming may influence patient food security status positively ${ }^{28}$ and have indirect benefits for patient health given the probability of their shared causal mechanisms. ${ }^{29}$ Greater and more direct benefits to patient health may be realized, though, if pantry visits result in greater access to and intake of nutritious foods, such as produce, given the well-documented relationship between diet and health. Future research should explore qualitatively the patient perceptions and related factors that may be driving differences in program use by diagnoses and other characteristics. Research should also examine the sustainability of referral-based programs in facilitating patients' use of food pantries and the impact of such use on food security, diet-related chronic disease management, and long-term health outcomes. ${ }^{10}$

\section{Practice Implications}

Our key finding - higher rates of food pantry use by patients with prior pantry experience - suggests that achieving broad impacts from this food referral program could require changes that encourage many patients to frequent the food pantry, as opposed to getting a select and already-engaged few to visit many times. ${ }^{30}$ Supporting more widespread and regular utilization may require a tailored referral, such as adding 1 additional question to the food insecurity screening process (eg, "Have you used a food pantry in the last year?"), so that potential first-time users could be flagged for an additional educational step or be provided other resources (eg, Lyft or Uber credits, or bus passes) to address barriers to food pantry access. In 
addition, reminding patients about the opportunity to visit the pantry, such as through text message reminders or secure messages via the patient's online health portal, could help encourage patients to use the community food resource. Reminders could also take place during a patient's future encounters with a health care provider. However, food referral program utilization data are not typically integrated back into the patient's EHR. This closed-loop referral approach remains an important next step to support utilization of food referral programs and to facilitate stronger integration between and mutual benefit for health care and communitybased organizations. Moving forward, it remains critical to engage the community of potential MOF participants in the refinement of the program. Application of community-based participatory research to this issue will be essential to learn from those eligible for the program and co-design solutions that may be most effective in increasing utilization in the short term and yield improvement in health outcomes and equity in the long term. ${ }^{31}$

\section{Limitations}

Our findings should be interpreted with a few key limitations in mind. First, the results reported here represent findings from an evaluation with a relatively limited sample size and period. As with any innovative programs, there were challenges experienced throughout the roll-out of the MOF that may have impacted the consistency of screening patients for food insecurity and providing eligible patients with a referral to the program. These challenges-and the self-selection inherent to programs like these that reach only those who have had a clinical encounter-may have resulted in selection bias for patients referred to the program that could skew results toward a greater percentage of visitors than in the general target population. Related, while only a small number of patients declined the referral, we did not document the reason for this decline. Related research suggests that stigma associated with the use of food pantries may create a barrier to access. ${ }^{32}$ Explaining why individuals might decline the referral remains an important issue to reach a broad population. Second, the focus of this analysis remained on MOF utilization rather than clinical endpoints, such as changes in BMI, BP, or HbA1C. These more distal outcomes of the program are critical to examine project effectiveness.
Third, our data spans the onset of the Coronavirus disease 2019 (COVID-19) pandemic. The COVID-19 pandemic has resulted in a significant rise in food insecurity due to the economic effects of the mitigation efforts. ${ }^{33}$ The impact of this exogenous shock are multifaceted and complex: while potential employment loss could have increased food insecurity status and need for food resources, social distancing measures put in place to mitigate the spread of COVID-19 may have impacted food pantry operations, patient willingness to visit the pantries, and transportation options available to interested patients. While these factors were not measured in our analysis, monitoring the impact of COVID-19 on food pantry utilization and related health outcomes for food-insecure patients with chronic conditions is an important and ongoing area of attention for researchers.

\section{Conclusions}

Our findings demonstrate that a clinic-based referral program at an AMC has the potential to increase the use of food pantries by food-insecure patients with chronic conditions. Health care providers can play an important role in connecting patients to community-based resources as a potential means to improve patient food security status and dietrelated health outcomes via increased access to food and intake of a health-promoting diet.

The authors would like to thank Seth Scarborough for assistance with data management.

To see this article online, please go to: http://jabfm.org/content/ 34/6/1174.full.

\section{References}

1. Anderson SA. Core indicators of nutritional state for difficult-to-sample populations. J Nutr 1990; 120:1555-600.

2. Palakshappa D, Speiser JL, Rosenthal GE, Vitolins $\mathrm{MZ}$. food insecurity is associated with an increased prevalence of comorbid medical conditions in obese adults: NHANES 2007-2014. J Gen Intern Med 2019;34:1486-93.

3. Gundersen C, Kreider B. Bounding the effects of food insecurity on children's health outcomes. J Health Econ 2009;28:971-83.

4. Holben DH, Pheley AM. Diabetes risk and obesity in food-insecure households in rural Appalachian Ohio. Prev Chronic Dis 2006;3:A82.

5. Seligman HK, Davis TC, Schillinger D, Wolf MS. Food insecurity is associated with hypoglycemia 
and poor diabetes self-management in a lowincome sample with diabetes. J Health Care Poor Underserved 2010;21:1227-33.

6. Seligman HK, Laraia BA, Kushel MB. Food insecurity is associated with chronic disease among lowincome NHANES participants. J Nutr 2010;140: 304-10.

7. RTI International. Current and prospective scope of hunger and food security in America: a review of current research. 2014. Available at: https://www. rti.org/sites/default/files/resources/full_hunger_ report_final_07-24-14.pdf. Accessed April 8, 2021.

8. An R, Wang J, Liu J, Shen J, Loehmer E, McCaffrey J. A systematic review of food pantrybased interventions in the USA. Public Health Nutr 2019;22:1704-16.

9. Bazerghi C, McKay FH, Dunn M. The role of food banks in addressing food insecurity: a systematic review. J Community Health 2016;41:732-40.

10. Cheyne K, Smith M, Felter EM, et al. Peer reviewed: food bank-based diabetes prevention intervention to address food security, dietary intake, and physical activity in a food-insecure cohort at high risk for diabetes. Prev Chronic Dis 2020;17: E04.

11. Rediger K, Miles DB. Clinical-community partnerships to reduce food insecurity among high-need, high-cost Medicaid patients. Ann Intern Med 2018;169:490-1.

12. Henize AW, Beck AF, Klein MD, Adams M, Kahn RS. A road map to address the social determinants of health through community collaboration. Pediatrics 2015;136:e993-e1001.

13. Berkowitz SA, Terranova J, Hill C, et al. Meal delivery programs reduce the use of costly health care in dually eligible Medicare and Medicaid beneficiaries. Health Aff (Millwood) 2018;37: 535-42.

14. De Marchis EH, Torres JM, Benesch T, et al. Interventions addressing food insecurity in health care settings: a systematic review. Ann Fam Med 2019;17:436-47.

15. Stenmark SH, Steiner JF, Marpadga S, Debor M, Underhill K, Seligman H. Lessons learned from implementation of the food insecurity screening and referral program at Kaiser Permanente Colorado. Perm J 2018;22:18-093.

16. Runkle NK, Nelson DA. The silence of food insecurity: disconnections between primary care and community organizations. J Patient Cent Res Rev 2021;8:31-8.

17. Clark A, Walker DM, Headings A. Addressing food insecurity in clinical care: lessons from the MidOhio farmacy experience. Health Affairs Blog. Jan 3, 2020. Available at: https://www.healthaffairs.org/ do/10.1377/hblog20191220.448706/full/.
18. Kaiser ML, Carr JK, Fontanella S. A tale of two food environments: differences in food availability and food shopping behaviors between food insecure and food secure households. J Hunger Environ Nutr 2019;14:297-317.

19. Feeding America. Map the Meal Gap 2020: Overall food insecurity in Franklin County, 2017. 2020. Available at: https://map.feedingamerica.org/county/ 2017/overall/ohio/county/franklin. Accessed December $10,2020$.

20. National Committee for Quality Assurance. HEDIS measures and technical resources: comprehensive diabetes care. 2020. Available at: https:// www.ncqa.org/hedis/measures/comprehensivediabetes-care/. Accessed December 10, 2020.

21. Carey RM, Calhoun DA, Bakris GL, American Heart Association Professional/Public Education and Publications Committee of the Council on Hypertension; Council on Cardiovascular and Stroke Nursing; Council on Clinical Cardiology; Council on Genomic and Precision Medicine; Council on Peripheral Vascular Disease; Council on Quality of Care and Outcomes Research; and Stroke Council, et al. Resistant hypertension: detection, evaluation, and management: a scientific statement from the American Heart Association. Hypertension 2018;72:e53-e90.

22. Hager ER, Quigg AM, Black MM, et al. Development and validity of a 2-item screen to identify families at risk for food insecurity. Pediatrics 2010;126:e26-32-e32.

23. Economic Research Service, United States Department of Agriculture. U.S. household food security survey module: three-stage design, with screeners. 2012. Available at: https://www.ers.usda. gov/media/8271/hh2012.pdf. Accessed Oct 27, 2019.

24. Ginsburg ZA, Bryan AD, Rubinstein EB, et al. Unreliable and difficult-to-access food for those in need: A qualitative and quantitative study of urban food pantries. J Community Health 2019;44:16-31.

25. De Marchis EH, Hessler D, Fichtenberg C, et al. Assessment of social risk factors and interest in receiving health care-based social assistance among adult patients and adult caregivers of pediatric patients. JAMA Netw Open 2020;3:e2021201-e2021201.

26. Zeigler BP, Redding SA, Leath BA, Carter EL. Pathways community HUB: a model for coordination of community health care. Popul Health Manag 2014;17:199-201.

27. Page-Reeves J, Kaufman W, Bleecker $M$, et al. Addressing social determinants of health in a clinic setting: the WellRx pilot in Albuquerque, New Mexico. J Am Board Fam Med 2016;29:414-8.

28. Aiyer JN, Raber M, Bello RS, et al. A pilot food prescription program promotes produce intake and decreases food insecurity. Translational Behavioral Med 2019;9:922-30. 
29. Leonard T, Hughes AE, Donegan C, Santillan A, Pruitt SL. Overlapping geographic clusters of food security and health: where do social determinants and health outcomes converge in the US? SSM Popul Health 2018;5:160-70.

30. Lantz PM. "Super-utilizer" interventions: what they reveal about evaluation research, wishful thinking, and health equity. The Milbank Quarterly 2020;98:

31. Wallerstein N, Duran B, Oetzel JG, Minkler M. Community-based participatory research for bealtb: advancing social and bealth equity. John Wiley \& Sons; 2017.

32. Purdam K, Garratt EA, Esmail A. Hungry? Food insecurity, social stigma and embarrassment in the UK. Sociology 2016;50:1072-88.

33. Feeding America. The impact of the coronavirus on food insecurity in 2020. 2020. Available at: https:// www.feedingamerica.org/sites/default/files/202010/Brief_Local\%20Impact_10.2020_0.pdf. Accessed Oct 30, 2020. 